\title{
Spectral Functions for the Quark-Meson Model Phase Diagram from the Functional Renormalization Group
}

\author{
Ralf-Arno Tripolt, ${ }^{1}$ Nils Strodthoff, ${ }^{2}$ Lorenz von Smekal, ${ }^{1,3}$ and Jochen Wambach ${ }^{1,4}$ \\ ${ }^{1}$ Theoriezentrum, Institut für Kernphysik, Technische Universität Darmstadt, 64289 Darmstadt, Germany \\ ${ }^{2}$ Institut für Theoretische Physik, Ruprecht-Karls-Universität Heidelberg, 69120 Heidelberg, Germany \\ ${ }^{3}$ Institut für Theoretische Physik, Justus-Liebig-Universität Giessen, 35392 Giessen, Germany \\ ${ }^{4}$ GSI Helmholtzzentrum für Schwerionenforschung GmbH, 64291 Darmstadt, Germany
}

\begin{abstract}
We present a method to obtain spectral functions at finite temperature and density from the Functional Renormalization Group. Our method is based on a thermodynamically consistent truncation of the flow equations for 2-point functions with analytically continued frequency components in the originally Euclidean external momenta. For the uniqueness of this continuation at finite temperature we furthermore implement the physical Baym-Mermin boundary conditions. We demonstrate the feasibility of the method by calculating the mesonic spectral functions in the quark-meson model along the temperature axis of the phase diagram, and at finite quark chemical potential along the fixed-temperature line that crosses the critical endpoint of the model.
\end{abstract}

PACS numbers: 12.38.Aw, 12.38.Lg, 11.10.Wx, 11.30.Rd

Keywords: spectral function, analytic continuation, QCD phase diagram, chiral phase transition

\section{INTRODUCTION}

Dynamic properties of strongly interacting matter are of fundamental interest to many theoretical as well as experimental studies in different branches of physics [1 7 ]. Spectral functions, for example, encode information on the particle spectrum as well as collective excitations and serve as input for the calculation of transport coefficients like the shear viscosity $[8,9$. However, the calculation of such real-time observables at finite temperature and density represents an inherently difficult problem.

On one hand, in the limit of vanishing external momenta, in which all information about $n$-point functions is encoded in the effective potential, nonperturbative methods are required even for equilibrium thermodynamics. Although mean-field calculations might capture the gross features of a phase diagram, quantitative predictions and correct descriptions of critical phenomena, e.g. by nontrivial critical exponents, require the proper inclusion of fluctuations. QCD thermodynamics at vanishing or nearly vanishing baryon density can be studied systematically with Monte-Carlo simulations on the lattice. In the region of higher baryon densities, however, the fermion sign-problem arises as an additional difficulty in Lattice QCD [10. This is one motivation for complementary approaches based on functional continuum methods which allow to include finite chemical potentials essentially without further complications. The particular one used in this work is the Functional Renormalization Group (FRG), see [11 17] for reviews.

On the other hand, a technical difficulty that arises already in the vacuum and which is common to all Euclidean approaches to Quantum Field Theory is the need to analytically continue from imaginary to real time for dynamic processes especially with timelike momentum transfer. This is even more so at finite temperature where these continuations are based on data at discrete Matsubara frequencies and hence require additional bound- ary conditions for uniqueness 18, 19. Even with the analytic structure completely fixed, however, the reconstruction of spectral functions from discrete numerical (i.e., noisy) data on Euclidean correlation functions, for example, is an ill-posed inverse problem. Maximum entropy methods (MEM) 20, 21, Padé approximants [22, or very recently also a standard Tikhonov regularization [23] have been proposed to deal with this problem. They all work best at low temperatures when the density of Matsubara modes is sufficiently large, but they all break down when the Euclidean input data is not sufficiently dense and precise.

Therefore any approach that can deal with the analytic continuation explicitly is highly desirable. Such alternative approaches were proposed in 24, 25] and 26] to involve an analytic continuation on the level of the flow equations themselves in order to provide correlation functions for timelike external momenta as the output of the calculations. In addition to its simplicity our approach enjoys a number of particular advantages: First of all, it is thermodynamically consistent in that the spacelike limit of zero external momentum in the 2-point correlation functions agrees with the curvature or screening masses as extracted from the thermodynamic grand potential 24]. Secondly, it satisfies the physical BaymMermin boundary conditions at finite temperature 18 whose implementation here proceeds essentially as in a simple one-loop calculation [27. Finally, although we focus on mesonic spectral functions in this work it can be extended to calculate also quark and gluonic spectral functions as an alternative to analytically continued Dyson-Schwinger equations (DSEs) [28, or to using MEM on Euclidean FRG 9 or DSE results 29 31.

A further extension would be to feed the full momentum dependence of the resulting 2-point functions back into the flow equation for the grand potential to solve this flow equation in an extended truncation, beyond the leading order derivative expansion. This would yield new 
3 and 4-point couplings to be used in the flow equations for the 2-point functions again, and this procedure could in principle be iterated to extend the consistency of the approach to their full momentum-dependence in this way.

Only such an iterative procedure will eventually provide a non-trivial width for resonance peaks in spectral functions. In this work we focus on the first iteration step of this procedure in which the momentum-dependent 2point functions are calculated on the basis of a given solution for the effective potential. Although the spacelike zero-momentum limits of the 2-point functions remain fixed to the effective potential, this is obviously not a fully self-consistent procedure for non-zero external momenta, and hence for the spectral functions at this point yet. One should keep in mind, however, that our calculations involve no prior knowledge of the momentum dependence or assumptions on the singularity structure of the propagators that one obtains.

In extension of previous results for the $O(4)$ linearsigma model in the vacuum [32, here we calculate mesonic spectral functions at finite temperature and with dynamical quarks at zero and finite density. This is done within the quark-meson model, which serves as a low energy effective model for QCD with $N_{\mathrm{f}}=2$ light quark flavors [33, 34]. In particular, it realizes the chiral symmetry breaking pattern of 2-flavor QCD and allows going beyond the mean-field approximation by including the fluctuations due to collective mesonic excitations. It has frequently been employed in model studies of the QCD phase diagram at finite temperature and density as a simple and intuitive replacement for more directly QCD based calculations [35 38, and it can systematically be improved towards full QCD by using appropriate input to model the influence of the gauge-field dynamics [39, 40]. For parameters that yield realistic quark and meson masses in the vacuum, the phase diagram of this model exhibits a first-order phase transition ending in a critical point and a crossover. It therefore allows for the study of critical regimes in the phase diagram as well as effects of chiral symmetry restoration.

This paper is organized as follows. In Sec. II we briefly introduce the FRG approach, especially for the quarkmeson model, and discuss our analytic continuation procedure. Results on thermodynamic observables, the phase diagram of the quark-meson model and mesonic spectral functions at finite temperature and chemical potential are shown in Sec. III. Finally, our summary and a brief outlook are provided in Sec. IV.

\section{THEORETICAL SETUP}

In this section we introduce the Functional Renormalization Group and its application to the quark-meson model as a chiral effective model for QCD. We derive the flow equations for the scale-dependent effective potential, and the corresponding ones for the pion and sigma meson 2-point functions together with our analytic continuation

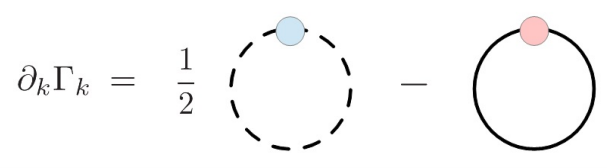

FIG. 1: (color online) Diagrammatic representation of the flow equation for the effective action. Dashed (solid) lines represent bosonic (fermionic) propagators and circles represent regulator insertions $\partial_{k} R_{k}$.

procedure for the originally discrete Matsubara frequencies as the zero-components of their external momenta at finite temperature.

\section{A. Functional Renormalization Group Approach to the Quark-Meson Model}

The FRG is a powerful tool for nonperturbative calculations in quantum field theory and statistical physics, see [11 17. It involves introducing an infrared (IR) regulator $R_{k}$ to suppress fluctuations from momentum modes with momenta below the associated renormalization group (RG) scale $k$. This regulator has then to be removed by taking $k$ from the ultraviolet (UV) cutoff scale $\Lambda$ down to zero. The central object in the approach pioneered by Wetterich [41] is the scale-dependent effective average action $\Gamma_{k}$ which thereby interpolates between the microscopic bare action at $k=\Lambda$ and the full quantum effective action for $k \rightarrow 0$. Its scale derivative is governed by an exact one-loop equation involving full scaleand field-dependent propagators which takes the form

$$
\partial_{k} \Gamma_{k}=\frac{1}{2} \mathrm{~S} \operatorname{Tr}\left[\partial_{k} R_{k}\left(\Gamma_{k}^{(2)}+R_{k}\right)^{-1}\right],
$$

where $\Gamma_{k}^{(2)}$ denotes the second functional derivative of the effective average action, and the supertrace includes internal and spacetime indices, the functional trace, typically in momentum space, and the minus sign with degeneracy factor for fermion loops. The flow equation for the effective average action is represented diagrammatically for bosonic and fermionic fields in Fig. 1 .

The calculation now proceeds by specifying an Ansatz for the effective average action $\Gamma_{k}$. For the quark meson model in the zeroth order derivative expansion, where only the effective potential carries a scale dependence, it reads

$$
\begin{array}{r}
\Gamma_{k}[\bar{\psi}, \psi, \phi]=\int d^{4} x\left\{\bar{\psi}\left(\not \partial+h\left(\sigma+i \vec{\tau} \cdot \vec{\pi} \gamma_{5}\right)-\mu \gamma_{0}\right) \psi\right. \\
\left.+\frac{1}{2}\left(\partial_{\mu} \phi\right)^{2}+U_{k}\left(\phi^{2}\right)-c \sigma\right\},(2)
\end{array}
$$

where $\phi_{i}=(\sigma, \vec{\pi})_{i}$ and $\phi^{2}=\sigma^{2}+\vec{\pi}^{2}$. The explicit symmetry breaking term $c \sigma$ accounts for a non-vanishing pion mass but does not enter the flow equation and is consequently only added to the full field-dependent potential at the IR scale. 
Given the flow equation in Eq. (1), one straightforwardly obtains flow equations for the 1-particle irreducible $n$-point functions upon taking $n$ functional derivatives of the flow equation for the effective average action with respect to the fields. In this work we are particularly interested in the flow equations for the pion and sigma meson 2-point functions which are represented diagrammatically in Fig. 2. The flow equations for $n$-point functions naturally involve up to $(n+2)$-point vertices as input which leads to an infinite tower of coupled equations. Hence, the numerical solution of such a system requires the use of truncations. In the following we neglect the momentum dependences of higher $n$-point vertex functions, which can be taken into account by more involved truncation schemes such as the BMW approximation [42, 43] for example, and treat them as the RG scale $k$ dependent couplings which we extract from the flowing effective potential. For now, the input 2-point functions in the loops on the right are also those obtained from the scale dependent average action at the leadingorder in the derivative expansion in Eq. (2). They are thus of tree-level form but contain the scale-dependent screening masses from the flow of the effective potential. We emphasize again that this scheme can be upgraded in the future to iteratively include the nontrivial momentum-dependences of the resulting 2-point functions as described in the introduction. The quark-meson 3 -point vertices in the flow equations for the 2-point functions remain scale-independent at this level,

$$
\Gamma_{\bar{\psi} \psi \phi_{i}}^{(2,1)}=h\left\{\begin{array}{ll}
1 & \text { for } i=0 \\
\mathrm{i} \gamma^{5} \tau^{i} & \text { for } i=1,2,3
\end{array},\right.
$$

while the mesonic 3 - and 4-point vertices are obtained in our truncation as

$$
\begin{aligned}
\Gamma_{\phi_{i} \phi_{j} \phi_{m}}^{(0,3)}= & 4 U_{k}^{\prime \prime}\left(\delta_{i j} \phi_{m}+\delta_{i m} \phi_{j}+\delta_{j m} \phi_{i}\right) \\
& +8 U_{k}^{(3)} \phi_{i} \phi_{j} \phi_{m}, \\
\Gamma_{\phi_{i} \phi_{j} \phi_{m} \phi_{n}}^{(0,4)}= & 4 U_{k}^{\prime \prime}\left(\delta_{i j} \delta_{m n}+\delta_{i n} \delta_{j m}+\delta_{j n} \delta_{i m}\right) \\
& +8 U_{k}^{(3)}\left(\delta_{i j} \phi_{m} \phi_{n}+\delta_{j m} \phi_{i} \phi_{n}+\delta_{m n} \phi_{i} \phi_{j}\right. \\
& \left.\quad+\delta_{j n} \phi_{i} \phi_{m}+\delta_{i n} \phi_{j} \phi_{m}+\delta_{i m} \phi_{j} \phi_{n}\right) \\
& +16 U_{k}^{(4)} \phi_{i} \phi_{j} \phi_{m} \phi_{n},
\end{aligned}
$$

where $U_{k}^{(n)}$ here denotes the $n$th derivative of the effective potential with respect to $\phi^{2}=\sigma^{2}+\vec{\pi}^{2}$.

The determination of higher $n$-point vertices using solely the input from the scale-dependent effective potential has the particular advantage that the flow equations for the 2-point functions evaluated at vanishing external momentum automatically reduce to the corresponding combinations of derivatives of the flow equation for the effective potential, as they must in a consistent truncation scheme based on a single generating object, which is in our case the effective potential. More explicitly, this implies that

$$
\begin{aligned}
& \partial_{k} \Gamma_{\pi, k}^{(0,2)}(p=0)=2 \partial_{k} U_{k}^{\prime}, \\
& \partial_{k} \Gamma_{\sigma, k}^{(0,2)}(p=0)=2 \partial_{k} U_{k}^{\prime}+4 \partial_{k} U_{k}^{\prime \prime} \phi^{2} .
\end{aligned}
$$

At finite temperature special care has to be taken with respect to the order of limits here. Once the frequency component $p_{0}$ of the external momentum is analytically continued, the static limit $\vec{p} \rightarrow 0$ with $p_{0}=0$ and the long-wavelength limit $p_{0} \rightarrow 0$ for $\vec{p}=0$ do not commute [24, 27, 44. The identities in (6) and (7) above are then understood as the spacelike static limit $\vec{p} \rightarrow 0$ of the lowest Matsubara mode $p_{0}=0$ before analytic continuation as they determine the static screening masses.

In this work we present the first step of the iteration procedure described above in which the 2-point functions are obtained by integrating their corresponding flow equations, see Fig. 2, built on the given solution for the effective potential as fixed input. While full selfconsistency can only be achieved by the iteration process, already the results for the 2-point functions after this first step show a non-trivial momentum dependence which is not restricted by any Ansatz. Note that unlike the iteration procedure for the effective potential, which is selfconsistent but requires only 2-point functions evaluated at Euclidean and hence spacelike external momenta as input, the calculations of real-time quantities such as spectral functions furthermore require 2-point functions evaluated at timelike external momenta which are obtained by analytic continuation as described below.

In the following we present the flow equations for the effective potential and the pion and sigma meson 2-point functions. The flow equation for the effective potential is obtained by evaluating Eq. (1) for constant fields and reads, with $N=4$ in our 2-flavor $O(4)$ case,

$$
\partial_{k} U_{k}=\frac{1}{2} I_{\sigma}^{(1)}+\frac{1}{2}(N-1) I_{\pi}^{(1)}-N_{\mathrm{c}} N_{\mathrm{f}} I_{\psi}^{(1)},
$$

where the loop functions $I_{\alpha}^{(i)}$ are defined as

$$
\begin{aligned}
I_{\alpha}^{(i)} & =\operatorname{Tr}_{q}\left[\partial_{k} R_{k}^{A}(q) G_{\alpha, k}^{i}(q)\right] \\
& \equiv \operatorname{Tr}_{q}\left[\partial_{k} R_{k}^{A}(q)\left(\Gamma_{\alpha, k}^{(2)}(q)+R_{k}^{A}(q)\right)^{-i}\right],
\end{aligned}
$$

with $\alpha \in\{\sigma, \pi, \psi\}, i>0$ and $A \in\{B, F\}$ chosen appropriately for bosonic and fermionic fields. The trace includes both a momentum integration and a trace over Dirac space for fermionic expressions while $G_{\alpha, k}(q)$ denotes the full Euclidean propagator at momentum $q$ and scale $k$ with implicit field variables replaced by their expectation values. In our numerical calculation we employ the three-dimensional analogue of the LPA-optimized regulator functions [45], namely

$$
\begin{aligned}
& R_{k}^{B}(q)=\left(k^{2}-\vec{q}^{2}\right) \theta\left(k^{2}-\vec{q}^{2}\right), \\
& R_{k}^{F}(q)=i \not q\left(\sqrt{\frac{k^{2}}{\vec{q}^{2}}}-1\right) \theta\left(k^{2}-\vec{q}^{2}\right),
\end{aligned}
$$




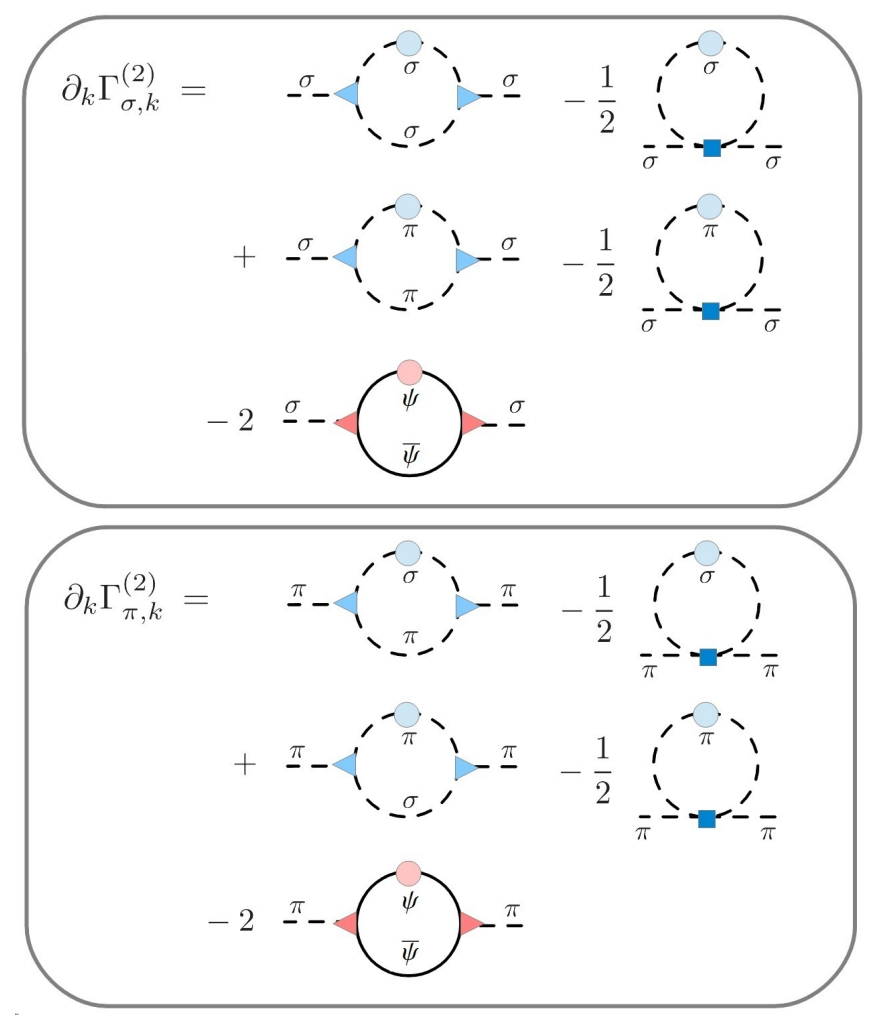

FIG. 2: (color online) Diagrammatic representation of the flow equation for the mesonic 2-point functions. Three-point vertices are represented by triangles, four-point vertices by squares.

for which the traces on the right hand side of the flow equations can be evaluated analytically which allows for a representation in terms of bosonic and fermionic occupation numbers, cf. Appendix B, a result that greatly alleviates the understanding of how to implement the correct analytic continuation.

The flow equations for the 2-point functions then read

$$
\begin{aligned}
& \partial_{k} \Gamma_{\pi, k}^{(2)}=\partial_{k} \Gamma_{\pi, k}^{(2), B}+\partial_{k} \Gamma_{\pi, k}^{(2), F}, \\
& \partial_{k} \Gamma_{\sigma, k}^{(2)}=\partial_{k} \Gamma_{\sigma, k}^{(2), B}+\partial_{k} \Gamma_{\sigma, k}^{(2), F},
\end{aligned}
$$

where the bosonic and the fermionic contributions are separately given by

$$
\begin{aligned}
\partial_{k} \Gamma_{\sigma, k}^{(2), B}= & J_{\sigma \sigma}^{B}\left(\Gamma_{\sigma \sigma \sigma}^{(0,3)}\right)^{2}+(N-1) J_{\pi \pi}^{B}\left(\Gamma_{\sigma \pi \pi}^{(0,3)}\right)^{2} \\
& -\frac{1}{2} I_{\sigma}^{(2)} \Gamma_{\sigma \sigma \sigma \sigma}^{(0,4)}-\frac{1}{2}(N-1) I_{\pi}^{(2)} \Gamma_{\sigma \sigma \pi \pi}^{(0,4)} \\
\partial_{k} \Gamma_{\pi, k}^{(2), B}= & \left(J_{\sigma \pi}^{B}+J_{\pi \sigma}^{B}\right)\left(\Gamma_{\sigma \pi \pi}^{(0,3)}\right)^{2}-\frac{1}{2} I_{\sigma}^{(2)} \Gamma_{\sigma \sigma \pi \pi}^{(0,4)} \\
& -\frac{1}{2} I_{\pi}^{(2)}\left(\Gamma_{\pi \pi \pi \pi}^{(0,4)}+(N-2) \Gamma_{\pi \pi \pi \tilde{\pi}}^{(0,4)}\right) \\
\partial_{k} \Gamma_{\sigma, k}^{(2), F}= & -2 N_{\mathrm{c}} N_{\mathrm{f}} J_{\sigma}^{F} \\
\partial_{k} \Gamma_{\pi, k}^{(2), F}= & -2 N_{\mathrm{c}} N_{\mathrm{f}} J_{\pi}^{F}
\end{aligned}
$$

with $\pi, \tilde{\pi} \in\left\{\pi_{1}, \pi_{2}, \pi_{3}\right\}$ and $\pi \neq \tilde{\pi}$. The bosonic and fermionic loop functions $J_{\alpha \beta}^{B} \equiv J_{\alpha \beta}^{B}(p)$ and $J_{\alpha}^{F} \equiv J_{\alpha}^{F}(p)$ for $\alpha \in\{\sigma, \pi\}$ at external momentum $p$ are defined as

$$
\begin{gathered}
J_{\alpha \beta}^{B}(p)=\operatorname{Tr}_{q}\left[\partial_{k} R_{k}^{B}(q) G_{\alpha, k}(q-p) G_{\beta, k}(q)^{2}\right], \\
J_{\alpha}^{F}(p)=\operatorname{Tr}_{q}\left[\partial_{k} R_{k}^{F}(q) G_{\psi, k}(q) \Gamma_{\bar{\psi} \psi \alpha}^{(2,1)}\right. \\
\left.G_{\psi, k}(q-p) \Gamma_{\bar{\psi} \psi \alpha}^{(2,1)} G_{\psi, k}(q)\right] .
\end{gathered}
$$

Explicit expressions for these loop functions can also be found in Appendix B.

\section{B. Analytic Continuation, Spectral Functions and UV Parameters}

As mentioned in the introduction, it is generally difficult to obtain timelike correlation functions from Euclidean results. One strategy is to attempt the inverse problem of reconstructing the corresponding spectral functions based on the known analyticity properties of local quantum field theory from the Euclidean input data, e.g. for 2-point functions. This can in principle be done with MEM which has become a standard procedure in lattice QCD, see, e.g., 21, 46 48. FRG studies have used both MEM [9] and Padé approximants [49 51 . for this purpose as well. Because it becomes an ill-posed problem when the input data is too noisy and incomplete, however, here we follow a different strategy as put forward in [24, 25, 32] and independently in [26]. It is based on the analytic continuation of the zero-component of the external momentum in the flow equation for the 2-point function itself. We thus directly solve flow equations for external frequencies in the complex plane. The analytic continuation is thereby somewhat subtle in general, and in particular at a finite RG scale $k$ where a generic regulator would introduce additional poles in the complex $q^{0}$-plane. Here we avoid this problem by using the purely spatial $3 d$-regulator functions (10) and (11) which do not introduce any spurious poles during the flow. The frequency component of the 4-momentum transfer remains unregulated and the corresponding Matsubara sums can be evaluated explicitly. The analytic continuation in the external frequency then proceeds formally as in a standard one-loop calculation in thermal field theory [27, 44]. The downside of this approach of course is that the $3 d$ regulators explicitly break the Euclidean $O(4)$ invariance. The effects of this have been assessed for the linear sigma model at $T=0$ and found to be negligible for Euclidean momenta up to several hundred MeV [32. While some residual frame dependence was observed in the spectral functions, however, their general shape and characteristic features such as particle poles and thresholds still remained unaffected by this breaking of Lorentz invariance in the timelike domain. We therefore expect that it should be safe to use the $3 d$ regulators here as well, especially in the rest frame of the thermal medium.

Explicitly, we employ the following two-step procedure for the analytic continuation from imaginary to real time: Once the sum over the Matsubara frequencies in the flow 
equation for the 2-point function is performed, we first exploit the periodicity of the bosonic and fermionic occupation numbers along the imaginary direction of the complex energy plane, i.e. with respect to discrete Euclidean external Matsubara modes $p_{0}=2 n \pi T$,

$$
n_{B, F}\left(E+\mathrm{i} p_{0}\right) \rightarrow n_{B, F}(E),
$$

cf. Appendix B. In the second step, the retarded 2-point functions are then obtained from their Euclidean counterparts via the analytic continuation

$$
\Gamma^{(2), R}(\omega, \vec{p})=-\lim _{\epsilon \rightarrow 0} \Gamma^{(2), E}\left(p_{0}=-\mathrm{i}(\omega+\mathrm{i} \epsilon), \vec{p}\right) .
$$

This substitution of the discrete Euclidean external $p_{0}$ by the continuous real frequency $\omega$ (with small imaginary part $\epsilon$ ) is done explicitly within the flow equation, before the integration of the RG scale $k$. Because of the one-loop structure of the flow equations together with the unregulated Matsubara sums, the correctness of this analytic continuation in the complex frequency plane follows directly from the corresponding one-loop formulae for the polarization functions in thermal field theory [27]. As in the one-loop examples, one therefore verifies that for the physically correct Baym-Mermin boundary conditions [18] it is crucial to follow these two steps in this order, i.e. first exploit the periodicity of the occupation numbers and then continue $p_{0}$ with $p_{0} \rightarrow-\mathrm{i}(\omega+i \epsilon)$.

In our numerical implementation we do not take the limit $\epsilon \rightarrow 0$ but keep a small but finite value of $\epsilon=1 \mathrm{MeV}$. Moreover, we consider only the special case in which the spatial external momentum components vanish, $\vec{p}=0$. Finally, the spectral function is given by the discontinuity of the propagator and can hence be expressed in terms of the imaginary part of the retarded propagator as,

$$
\rho(\omega)=-\frac{1}{\pi} \frac{\operatorname{Im} \Gamma^{(2), R}(\omega)}{\left(\operatorname{Re} \Gamma^{(2), R}(\omega)\right)^{2}+\left(\operatorname{Im} \Gamma^{(2), R}(\omega)\right)^{2}} .
$$

To numerically solve the flow equation for the effective potential, Eq. (8), and for the real and imaginary parts of the 2-point functions, Eqs. (12) and (13), we have to specify the model parameters and provide initial conditions. For the effective potential we assume the following shape in the UV,

$$
U_{\Lambda}\left(\phi^{2}\right)=\frac{1}{2} m_{\Lambda}^{2} \phi^{2}+\frac{1}{4} \lambda_{\Lambda}\left(\phi^{2}\right)^{2},
$$

while for the sigma and pion 2-point function we employ

$$
\begin{aligned}
& \Gamma_{\sigma, \Lambda}^{(2), R}(\omega)=-\omega^{2}+2 U_{\Lambda}^{\prime}+4 \phi^{2} U_{\Lambda}^{\prime \prime} \\
& \Gamma_{\pi, \Lambda}^{(2), R}(\omega)=-\omega^{2}+2 U_{\Lambda}^{\prime} .
\end{aligned}
$$

The model parameters are chosen to obtain phenomenologically reasonable values for the quark and meson masses in the IR. Using the values listed in Table II. we obtain at vanishing temperature and chemical potential a quark mass of $m_{\psi}=299 \mathrm{MeV}$, a pion mass of $m_{\pi}=138 \mathrm{MeV}$ and a sigma meson mass of

\begin{tabular}{c|c|c|c|c}
$\Lambda / \mathrm{MeV}$ & $m_{\Lambda} / \Lambda$ & $\lambda_{\Lambda}$ & $c / \Lambda^{3}$ & $h$ \\
\hline 1000 & 0.794 & 2.00 & 0.00175 & 3.2
\end{tabular}

TABLE I: Employed parameter values.

$m_{\sigma}=509 \mathrm{MeV}$. The chiral order parameter, to be identified with the pion decay constant, takes a value of $\sigma_{0} \equiv f_{\pi}=93.5 \mathrm{MeV}$. We note here that if the temperature gets too large compared to the UV cutoff scale, in our case of $T_{\max } \approx \Lambda / 2 \pi \approx 170 \mathrm{MeV}$, the assumption of a temperature-independent effective action in the UV breaks down. If one wants to extend the accessible temperature range for a fixed UV cutoff, the results have to be supplemented by perturbative input [52 54. We checked, however, that this limitation does not change the qualitative features discussed in the following.

\section{Numerical procedure}

The flow equation for the effective potential, Eq. (8), is solved by discretizing the effective potential on a grid in field space, see 34 for details, which turns this partial differential equation into a set of ordinary differential equations which are then integrated from the UV scale $\Lambda$ to some IR scale $k_{\text {IR }}$. This process gets increasingly expensive the smaller the infrared scale at which the flow is stopped. Here it is chosen as $k_{\mathrm{IR}}=40 \mathrm{MeV}$.

The flow equations for the real and imaginary parts of the mesonic 2-point functions are subsequently solved at the fixed global minimum of the effective potential in the IR, i.e. for $\phi=\sigma_{0}$. Typical problems that arise in this process are cutoff-effects due to the 'abrupt' start and end of the integration of the flow equations. These cutoff effects, which can produce negative values for the spectral functions, can be corrected by using extrapolations of the scale-dependent meson masses and the necessary derivatives of the effective potential to integrate the flow equations for the 2-point functions to even smaller, and larger, scales. Another difficulty stems from the fact that the flow equations for the 2-point functions are numerically highly sensitive to the scale-dependence of the meson masses, $m_{\sigma}(k)$ and $m_{\pi}(k)$. At the critical point, for example, the sigma mass undergoes drastic changes over a small $k$-range which hampers the calculation of spectral functions at certain values of $\omega$, which are excluded from our discussion in the following. A similar problem arises at higher temperatures, $T \gtrsim 50 \mathrm{MeV}$, where terms corresponding to the process of two pions going into a sigma meson, that are proportional to the derivative of bosonic occupation numbers, cf. Eq. (B15), are no longer suppressed and can lead to negative values of the pion spectral function in a certain $\omega$ range. Where necessary, we therefore use an interpolation of the scale-dependent meson masses between $\Lambda$ and $k_{\mathrm{IR}}$, thus eliminating numerical fluctuations and providing a smooth shape of $m_{\sigma}(k)$ and $m_{\pi}(k)$, to integrate the aforementioned terms. 


\section{RESULTS}

\section{A. Phase diagram and screening masses}

We first briefly discuss the phase diagram obtained from the quark-meson model as it serves as input for the calculation of the 2-point functions here. It is shown in Fig. 3 and displays the typical shape 34] found in quark-meson model calculations beyond the mean-field approximation with a critical endpoint at $\mu=293 \mathrm{MeV}$ and $T=10 \mathrm{MeV}$.

In addition, it is also instructive to consider the temperature- and chemical potential-dependence of the meson screening masses as they will be used to identify thresholds in the spectral functions. The meson screening masses, $m_{\sigma}$ and $m_{\pi}$, can be extracted from the effective potential, cf. Eq. (B6), and are shown in Fig. 4 together with the quark mass $m_{\psi}$ and the order parameter $\sigma_{0}$ as a function of temperature at $\mu=0 \mathrm{MeV}$ (left panel) and as a function of chemical potential at $T=10 \mathrm{MeV}$ (right panel). In particular, one way of defining a pseudocritical temperature for the crossover is by using the maximum of the chiral susceptibility $\chi_{\sigma} \equiv 1 / m_{\sigma}^{2}$, see [55, 56] and 54] for a comparison of different definitions of pseudo-critical temperatures, which leads to value of $T_{c} \approx 175 \mathrm{MeV}$ in our case. At higher temperatures the sigma and pion mass degenerate, while the order parameter drastically decreases, indicating the progressive restoration of chiral symmetry.

At vanishing temperature the Silver Blaze property [57] ensures that the partition function and correspondingly all thermodynamic observables remain independent of the chemical potential $\mu$ until it exceeds the constituent quark mass in the vacuum or until it reaches a first order transition whichever occurs first. This is approximately true here as well with the exception of the observed decrease in the sigma mass above $250 \mathrm{MeV}$ which is a finite temperature effect. At $T=10 \mathrm{MeV}$ and $\mu \approx 293 \mathrm{MeV}$, i.e. near the critical endpoint, the sigma mass suddenly further drops significantly, as expected near a second order phase transition. At even higher values of the chemical potential the meson masses become degenerate again, similar to the high temperature case.

\section{B. Spectral functions}

We now turn to the discussion of results for the sigma and pion spectral functions, $\rho_{\sigma}(\omega)$ and $\rho_{\pi}(\omega)$. They are shown in Fig. 5 as a function of external energy $\omega$ at different values of temperature and chemical potential. The inserted numbers refer to different processes contributing to the spectral functions via the corresponding diagrams shown in Fig. 2. The sigma spectral function is affected by the processes $\sigma^{\prime} \rightarrow \sigma \sigma(1), \sigma^{\prime} \rightarrow \pi \pi(2)$ and $\sigma^{\prime} \rightarrow \bar{\psi} \psi$ (3), where primes denote off-shell correlations with energy $\omega$. The relevant processes for the pion spectral function are $\pi^{\prime} \rightarrow \sigma \pi(4), \pi^{\prime} \pi \rightarrow \sigma(5)$ and $\pi^{\prime} \rightarrow \bar{\psi} \psi(6)$. In

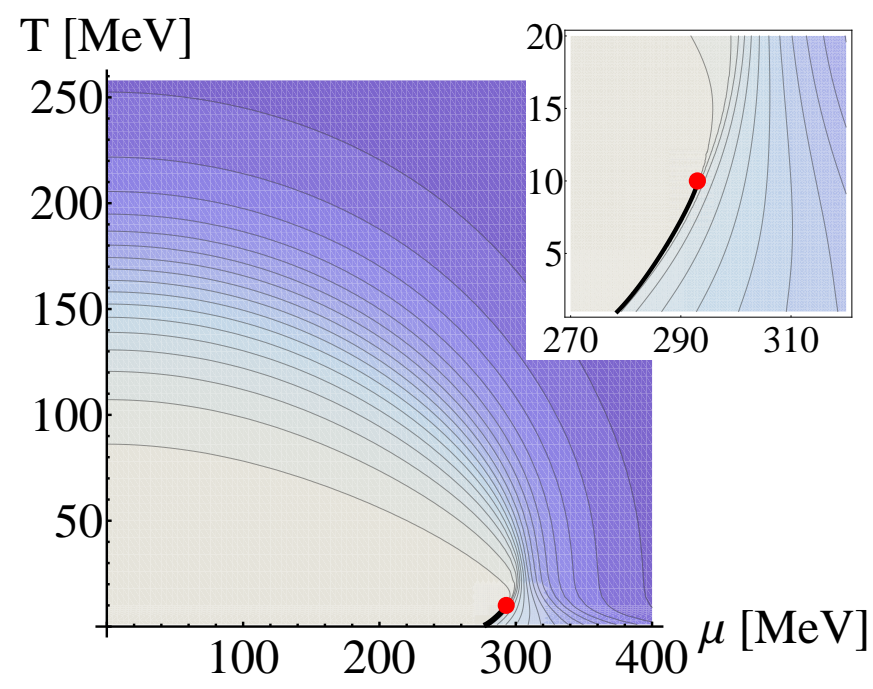

FIG. 3: (color online) The phase diagram of the quark-meson model, obtained by using the parameter set in Tab. I is illustrated by a contour plot of the magnitude of the chiral order parameter, $\sigma_{0} \equiv f_{\pi}$, vs. quark chemical potential $\mu$ and temperature $T$. The order parameter decreases towards higher $\mu$ and $T$, indicated by darker color. The critical endpoint is denoted by a red dot, connected to the black first-order transition line.

our truncation with momentum-independent vertices the mesonic tadpole diagrams only give rise to $\omega$-independent contributions to the spectral functions.

At $T=10 \mathrm{MeV}$ and $\mu=0 \mathrm{MeV}$ the spectral functions closely resemble the vacuum structure already observed in previous studies, cf. [25, 32]. The pion spectral function exhibits a peak close to $\omega=100 \mathrm{MeV}$, originating from a zero-crossing of the real part of $\Gamma_{\pi}^{(2), R}(\omega)$ to be identified with the pion pole mass, cf. Appendix A for a brief account of the real and imaginary part of the sigma and pion 2-point-functions. The corresponding screening mass, determined from the effective potential, is found to be $m_{\pi}=138 \mathrm{MeV}$, cf. Fig. 4, and thus considerably larger than the pole mass. Discrepancies between the pole and screening masses were also observed in [24] and less pronounced in purely bosonic models within similar truncations [58, 59], and are expected to decrease with an increased momentum dependence of the truncation. For external energies larger than $2 m_{\psi}$ the decay of an (offshell) pion into two quarks becomes energetically possible, giving rise to an increase of the imaginary part of $-\Gamma_{\pi}^{(2), R}(\omega)$ and thus also to the pion spectral function for $\omega \geq 2 m_{\psi} \approx 600 \mathrm{MeV}$. In addition, the process $\pi^{\prime} \rightarrow \sigma \pi$ leads to modifications of the pion spectral function for $\omega \geq m_{\sigma}+m_{\pi} \approx 650 \mathrm{MeV}$, but its contribution is about a magnitude smaller than that of the quark decay and therefore not visible in Fig. 5. The remaining reaction affecting the pion spectral function, $\pi^{\prime} \pi \rightarrow \sigma$, is strongly suppressed at low temperatures, since it is proportional to terms involving the derivative of bosonic occupation numbers, cf. Eq. (B15). 

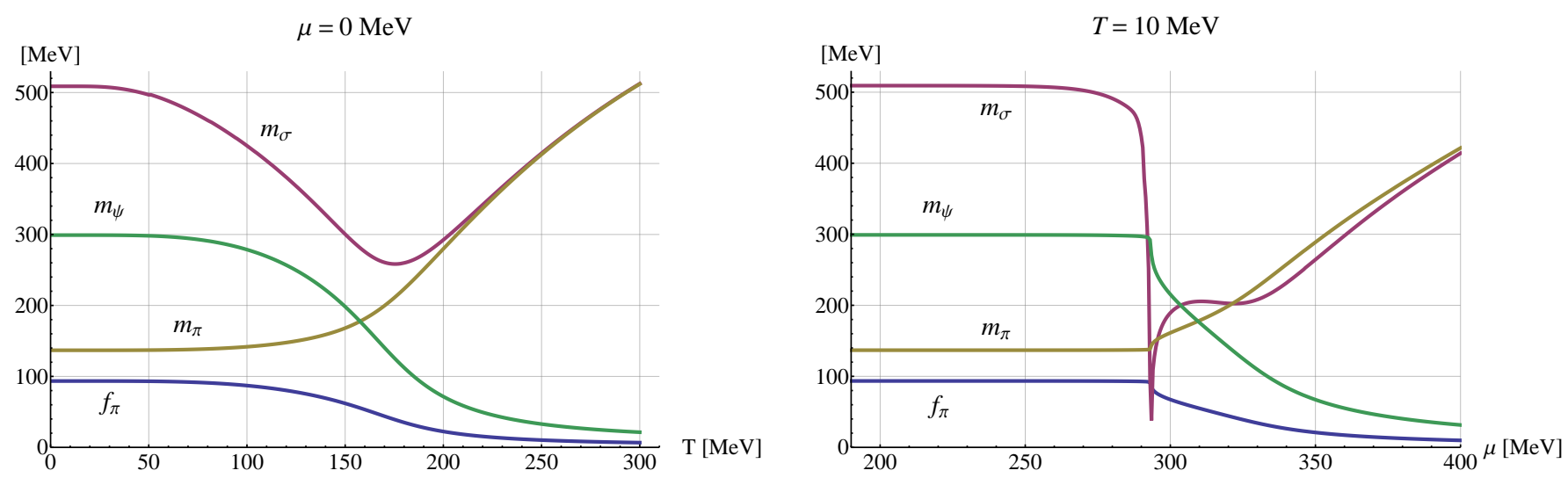

FIG. 4: (color online) The meson screening masses, the quark mass and the chiral order parameter, $\sigma_{0} \equiv f_{\pi}$, are shown vs. temperature $T$ at $\mu=0 \mathrm{MeV}$ (left panel), and vs. quark chemical potential $\mu$ at $T=10 \mathrm{MeV}$ (right panel).

The dominant channel affecting the sigma spectral function is the decay into two pions, $\sigma^{\prime} \rightarrow \pi \pi$, which is possible for $\omega \geq 2 m_{\pi}$. This process leads to a strong increase of the imaginary part of $\Gamma_{\sigma}^{(2), R}(\omega)$ at $\omega \gtrsim 275 \mathrm{MeV}$, even before the zero-crossing of the real part at $\omega \approx$ $340 \mathrm{MeV}$. At external energies larger than $2 m_{\psi}$ the $\sigma^{\prime} \rightarrow \bar{\psi} \psi$ channel opens up but yields only small contributions compared to the decay into two pions. The process $\sigma^{\prime} \rightarrow \sigma \sigma$ can only occur at external energies larger than $2 m_{\sigma}$, i.e. beyond $1 \mathrm{GeV}$, which are not shown in Fig. 5 .

When going to higher temperatures the process $\pi^{\prime} \pi \rightarrow \sigma$, which describes the scattering of an off-shell pion with a pion from the heat bath resulting in the formation of a sigma meson, becomes less suppressed and contributes to the pion spectral function for $\omega \leq$ $m_{\sigma}-m_{\pi}$. Since the difference between the meson and pion screening masses continuously decreases with higher temperature at $\mu=0 \mathrm{MeV}$, cf. Fig. 4, the maximum external energy up to which this process is possible is also shifted to smaller values, as is the corresponding bump of the pion spectral function, cf. Fig. 5 .

Another effect induced by the temperature dependence of the meson and quark masses is the emergence of a stable sigma meson at temperatures close to the crossover temperature, where neither the decay into two pions nor into two quarks is energetically possible. At $T=150 \mathrm{MeV}$ we therefore observe a pronounced peak in the sigma spectral function at $\omega \approx 280 \mathrm{MeV}$, originating from a zero-crossing of the real part of $\Gamma_{\sigma}^{(2), R}(\omega)$. When increasing the temperature further, the quarks become the lightest degrees of freedom considered here, providing decay channels for both the pion and the sigma meson down to small external energies and leading to a broadening of the pronounced peaks in the spectral functions.

At $T=250 \mathrm{MeV}$, the meson screening masses are nearly degenerate and have increased to about $400 \mathrm{MeV}$, thus shifting the threshold for decays into mesons to twice that value. Additionally, the quark mass has further decreased, to approximately $30 \mathrm{MeV}$, leading to a broad maximum in the almost degenerate sigma and pion spec- tral functions. These effects of chiral symmetry restoration agree qualitatively with other studies, e.g. 60] where mesonic spectral functions were studied at finite temperature within an $O(N)$ model using optimized perturbation theory.

The right column of Fig. 5 shows the sigma and pion spectral function at a fixed temperature of $T=10 \mathrm{MeV}$ and different values of the quark chemical potential, corresponding to points on a horizontal line in the phase diagram near the critical endpoint. We note that contributions to the pion spectral function arising from the process $\pi^{\prime} \pi \rightarrow \sigma$ are negligible at such low temperatures and are therefore not indicated in these figures. Over a wide range of chemical potentials the spectral functions remain basically unchanged, as expected from the aforementioned Silver Blaze property. Between $\mu=0$ and $\mu=200 \mathrm{MeV}$ the results are practically identical (compare the top left and top right panels in Fig. 5). When approaching the critical endpoint, however, especially the sigma spectral function undergoes significant changes.

At $\mu=292 \mathrm{MeV}$, i.e. only about $1 \mathrm{MeV}$ from the critical endpoint, the sigma screening mass has already dropped to about half its vacuum value, leading to a minimal energy for the $\sigma^{\prime} \rightarrow \sigma \sigma$ decay of $\omega \geq 2 m_{\sigma} \approx$ $540 \mathrm{MeV}$. Additionally, a pronounced sigma peak starts to develop at $\omega \approx 290 \mathrm{MeV}$, indicating the formation of a stable dynamical sigma meson, cf. Appendix A.

Even closer to the CEP, i.e. at $\mu=292.8 \mathrm{MeV}$, the threshold for the two-sigma decay has decreased to $\omega \approx$ $290 \mathrm{MeV}$ and thus occurs already at smaller energies than the $\sigma^{\prime} \rightarrow \pi \pi$ process. The sigma pole mass, given by the location of the sharp peak in the sigma spectral function, has also decreased considerably, taking a value of $m_{\sigma}^{p} \approx$ $140 \mathrm{MeV}$, still in very good agreement with its screening mass at this point.

At $\mu=292.97 \mathrm{MeV}$, however, the sigma pole mass has already decreased to $m_{\sigma}^{p} \approx 20 \mathrm{MeV}$, whereas the $\sigma^{\prime} \rightarrow \sigma \sigma$ threshold, as determined by the screening mass here, lags behind. Because the $\sigma$ screening mass changes very rapidly close to the endpoint, cf. Fig. 4, the differ- 

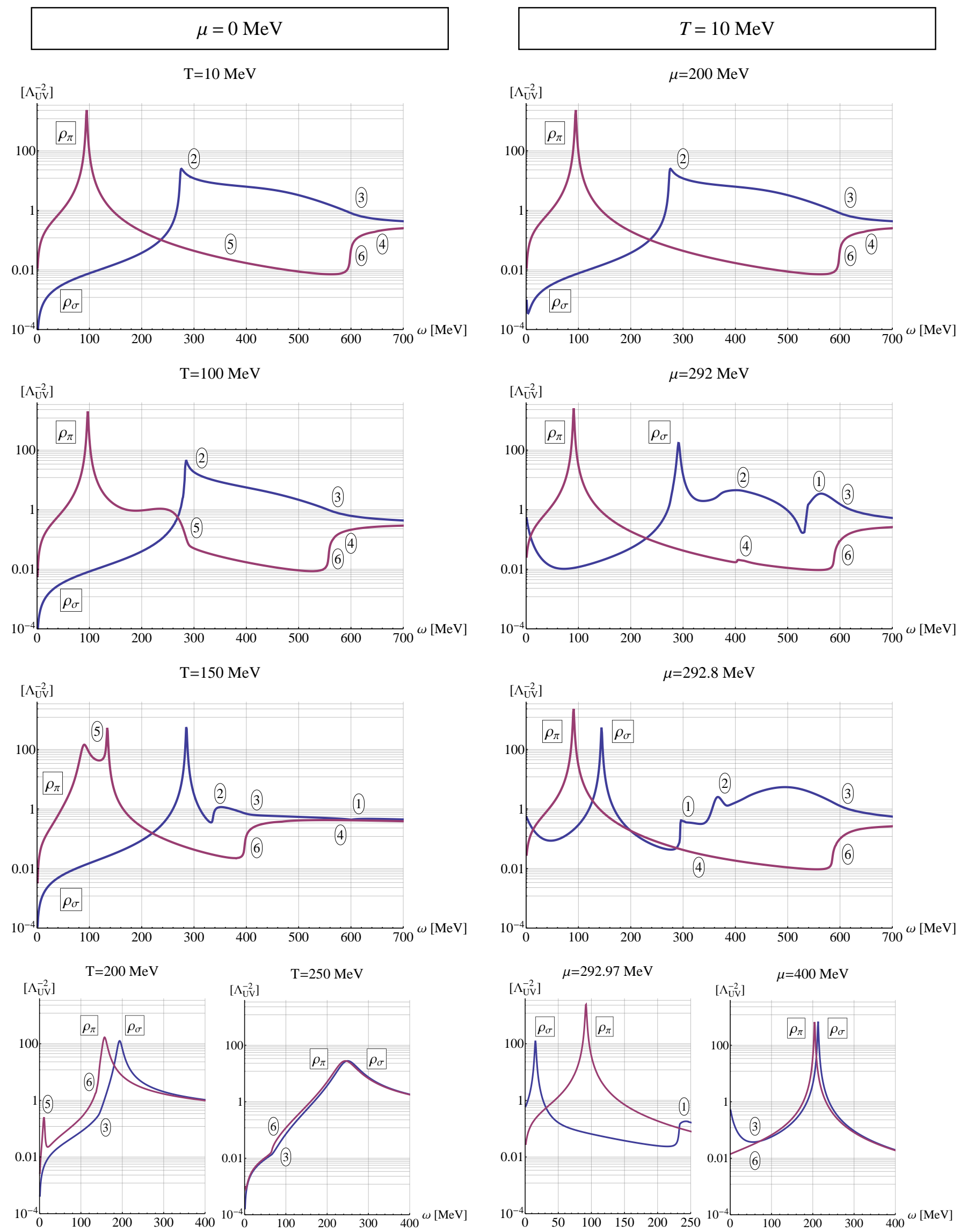

FIG. 5: (color online) Sigma and pion spectral function, $\rho_{\sigma}(\omega)$ and $\rho_{\pi}(\omega)$, are shown versus external energy $\omega$ at $\mu=0 \mathrm{MeV}$ but different $T$ (left column) and at $T=10 \mathrm{MeV}$ but different $\mu$ (right column). Inserted numbers refer to the different processes affecting the spectral functions at the so indicated values of $\omega .1: \sigma^{\prime} \rightarrow \sigma \sigma, 2: \sigma^{\prime} \rightarrow \pi \pi, 3: \sigma^{\prime} \rightarrow \bar{\psi} \psi, 4: \pi^{\prime} \rightarrow \sigma \pi, 5: \pi^{\prime} \pi \rightarrow \sigma$, 6: $\pi^{\prime} \rightarrow \bar{\psi} \psi$. See text for details. 
ence between the pole and half the $2 \sigma$-threshold is very sensitive to this remaining inconsistency in our present truncation and hence enhanced in this tiny region around the endpoint. It is manifest in our approach, however, that the pole mass and the screening mass of the sigma will both be zero precisely at the critical endpoint and hence identical again.

When increasing the chemical potential further, the sigma and pion spectral functions become degenerate, similar to the case of high temperatures, but with the difference that the spectral functions exhibit sharp peaks instead of broad resonances, indicating stable dynamical particles. We also observe a small increase of the sigma spectral function at small external energies which originates from the different prefactors arising in the definition of the fermionic loop functions $J_{\alpha}^{F}\left(p_{0}, k\right)$, cf. Eqs. (B13)-(B14), which in turn are due to the different Dirac structure of the quark-sigma and quark-pion vertices given in Eq. (3). This effect, however, is found to diminish when going to even higher values of chemical potential, as expected since the fermionic loop functions $J_{\sigma}^{F}\left(p_{0}, k\right)$ and $J_{\pi}^{F}\left(p_{0}, k\right)$ are identical in the limit of vanishing quark mass.

\section{SUMMARY AND OUTLOOK}

In this work we have presented a method for calculating spectral functions at finite temperature and quark chemical potential within the Functional Renormalization Group approach, based on previous studies in the vacuum 32. Our method involves an analytic continuation from imaginary to real frequencies on the level of the flow equations which realizes the physical Baym-Mermin boundary conditions. It is based on a thermodynamically consistent truncation of the flow equations for 2-point functions and allows to iteratively take into account the full momentum dependence of 2-point functions without need for assumptions on the analytic structure of the propagators in the future.

Results have been obtained for mesonic spectral functions of the quark-meson model at finite temperature and density. Our presentation concentrated on the temperature dependence at vanishing chemical potential and on the vicinity of the critical point. Thereby, effects of the following in-medium processes on the spectral functions have been discussed: $\pi^{\prime} \rightarrow \sigma \pi, \pi^{\prime} \pi \rightarrow \sigma$ and $\pi^{\prime} \rightarrow \bar{\psi} \psi$ for the pion spectral function, and $\sigma^{\prime} \rightarrow \sigma \sigma, \sigma^{\prime} \rightarrow \pi \pi$ and $\sigma^{\prime} \rightarrow \bar{\psi} \psi$ for the sigma spectral function. At low temperatures and small values of chemical potential the spectral functions closely resemble the vacuum structure observed in previous studies 32]. Near the critical endpoint in particular the decay of an off-shell sigma meson into two on-shell sigma mesons results in drastic changes of the sigma spectral function. At high temperatures and/or large values of the chemical potential a degeneration of the spectral functions was observed indicative of chiral symmetry restoration.
The results shown in this work represent a promising extension of the method for the calculation of spectral functions to finite temperature and chemical potential and can be improved in several ways. Apart from the extension to other spectral functions or the iteration procedure described above, another straightforward but important extension will be the inclusion of non-vanishing external spatial momenta. This will then also allow to use the method for calculations of transport coefficients such as the shear viscosity.

\section{Acknowledgments}

The authors thank Kazuhiko Kamikado and Jan Pawlowski for discussions and work on related subjects. This work was supported by the Helmholtz International Center for FAIR within the LOEWE initiative of the State of Hesse. L.v.S. is furthermore supported by the European Commission, FP-7-PEOPLE-2009-RG, No. 249203, N.S. by the grant ERC-AdG-290623, and R.-A.T. by the Helmholtz Research School for Quark Matter Studies, H-QM.

\section{Appendix A: Real and imaginary part of the 2-point function}

As described in Section II B the spectral function can be expressed in terms of the real and imaginary part of the retarded 2-point function, see Eq. (22). In this section we present results on the real and imaginary parts of the sigma and pion 2-point functions to further illustrate the behavior of the corresponding spectral functions discussed in Section III.

In Fig. 6 the real and imaginary part of the sigma and pion 2-point functions are plotted over the external energy for two representative cases also discussed in Section III, namely at $\mu=0 \mathrm{MeV}$ and $T=100 \mathrm{MeV}$ (left column) as well as at $\mu=292 \mathrm{MeV}$ and $T=10 \mathrm{MeV}$ (right column).

At vanishing chemical potential and a temperature of $100 \mathrm{MeV}$, the real part of the sigma 2-point function shows a zero-crossing at $\omega \approx 290 \mathrm{MeV}$. Since, however, the imaginary part takes on a non-zero value already for $\omega \geq 2 m_{\pi} \approx 280 \mathrm{MeV}$, due to the decay of a sigma meson into two pions, the zero-crossing of the real part does not give rise to a stable sigma state. At $\omega \geq 2 m_{\psi} \approx 560 \mathrm{MeV}$, also the decay into a quark and an anti-quark contributes to the imaginary part of the sigma 2-point function.

The real part of the pion 2-point function exhibits a zero-crossing at $\omega \approx 100 \mathrm{MeV}$, giving rise to a pronounced peak in the spectral function, cf. Fig. 5. since the imaginary part does not develop non-vanishing values until external energies around $260 \mathrm{MeV}$ from the $\pi^{\prime} \pi \rightarrow \sigma$ process. For $\omega \geq 2 m_{\psi}$, the quark decay channel and, for $\omega \geq m_{\sigma}+m_{\pi}$, also the decay into a pion and a sigma 

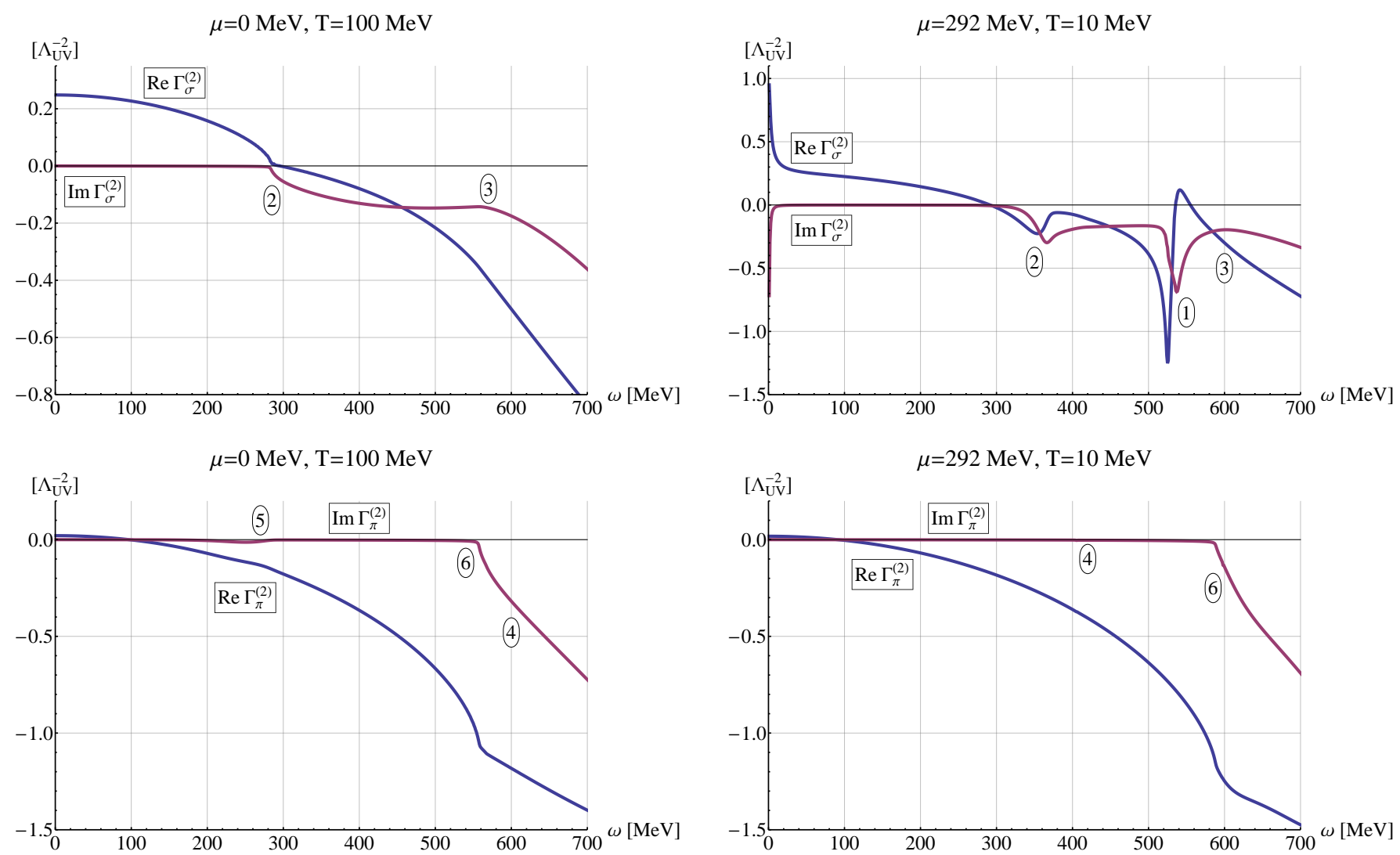

FIG. 6: (color online) The real and imaginary parts of the sigma and pion two-point functions, $\Gamma_{\sigma, \pi}^{(2), R}(\omega)$, are shown versus external energy $\omega$ at $\mu=0 \mathrm{MeV}$ and $T=100 \mathrm{MeV}$ (left column) as well as at $\mu=292 \mathrm{MeV}$ and $T=10 \mathrm{MeV}$ (right column), cf. Fig. 5 Inserted numbers refer to the different processes contributing to the 2-point functions at the so indicated values of $\omega .1: \sigma^{\prime} \rightarrow \sigma \sigma, 2: \sigma^{\prime} \rightarrow \pi \pi, 3: \sigma^{\prime} \rightarrow \bar{\psi} \psi, 4: \pi^{\prime} \rightarrow \sigma \pi, 5: \pi^{\prime} \pi \rightarrow \sigma, 6: \pi^{\prime} \rightarrow \bar{\psi} \psi$

meson give rise to large negative values of the imaginary part of the pion 2-point function.

At a chemical potential of $292 \mathrm{MeV}$ and a temperature of $10 \mathrm{MeV}$, i.e. near the critical endpoint, the real and imaginary part of the sigma 2-point function show large modifications from all decay channels, in particular from the decay of an off-shell sigma state into two sigma mesons, $\sigma^{\prime} \rightarrow \sigma \sigma$, which becomes energetically possible for $\omega \geq 2 m_{\sigma} \approx 520 \mathrm{MeV}$. At $\omega \approx 290 \mathrm{MeV}$ the real part has a zero-crossing while the imaginary part is still close to zero, giving rise to a pronounced peak in the spectral function, cf. Fig. 5 .

The real and imaginary part of the pion 2-point function on the other hand show only small changes compared to their vacuum shape, cf. 32 for a discussion within the $O(N)$ model. The zero-crossing of the real part near $\omega \approx 100 \mathrm{MeV}$ still gives rise to a stable pion while at $\omega \geq 2 m_{\psi} \approx 580 \mathrm{MeV}$ the decay into a quark-antiquark pair produces negative values of the imaginary part of the pion 2-point function.

\section{Appendix B: Threshold functions}

In this section we list the explicit expressions for the threshold functions appearing in the flow equations for the effective potential, Eq. (8), and the bosonic two-point functions, Eqs. (14)-(17), using the $3 \mathrm{~d}$ regulator functions from Eq. (10).

Explicitly, the loop functions $I_{\alpha}^{(1)}$ and $I_{\alpha}^{(2)}$ are given by

$$
\begin{aligned}
I_{\sigma, \pi}^{(1)} & =\frac{k^{4}}{6 \pi^{2}} \frac{1+2 n_{B}\left(E_{\sigma, \pi}\right)}{E_{\sigma, \pi}}, \\
I_{\psi}^{(1)} & =\frac{k^{4}}{3 \pi^{2}} \frac{1-n_{F}\left(E_{\psi}-\mu\right)-n_{F}\left(E_{\psi}+\mu\right)}{E_{\psi}}, \\
I_{\sigma, \pi}^{(2)} & =\frac{k^{4}}{6 \pi^{2}}\left(\frac{1+2 n_{B}\left(E_{\sigma, \pi}\right)}{2 E_{\sigma, \pi}^{3}}-\frac{n_{B}^{\prime}\left(E_{\sigma, \pi}\right)}{E_{\sigma, \pi}^{2}}\right),
\end{aligned}
$$

with the bosonic and fermionic occupation numbers

$$
n_{B}(E)=\frac{1}{e^{E / T}-1}, \quad n_{F}(E)=\frac{1}{e^{E / T}+1} .
$$

The effective quasi-particle energies read

$$
E_{\alpha}=\sqrt{k^{2}+m_{\alpha}^{2}}, \quad \alpha \in\{\pi, \sigma, \psi\},
$$

where the effective meson masses and the quark mass are obtained as

$$
m_{\pi}^{2}=2 U_{k}^{\prime}, \quad m_{\sigma}^{2}=2 U_{k}^{\prime}+4 U_{k}^{\prime \prime} \phi^{2}, \quad m_{\psi}^{2}=h^{2} \phi^{2} .
$$


The explicit expressions for the vertex functions appearing in Eqs. 14 -17 read

$$
\begin{aligned}
\Gamma_{\sigma \sigma \sigma}^{(0,3)} & =12 U_{k}^{\prime \prime} \phi+8 U_{k}^{(3)} \phi^{3}, \\
\Gamma_{\sigma \pi_{1} \pi_{1}}^{(0,3)} & =4 U_{k}^{\prime \prime} \phi, \\
\Gamma_{\sigma \sigma \sigma \sigma}^{(0,4)} & =12 U_{k}^{\prime \prime}+48 U_{k}^{(3)} \phi^{2}+16 U_{k}^{(4)} \phi^{4}, \\
\Gamma_{\pi_{1} \pi_{1} \pi_{2} \pi_{2}}^{(0,4)} & =4 U_{k}^{\prime \prime}, \\
\Gamma_{\pi_{1} \pi_{1} \pi_{1} \pi_{1}}^{(0,4)} & =12 U_{k}^{\prime \prime}, \\
\Gamma_{\sigma \sigma \pi_{1} \pi_{1}}^{(0,4)} & =4 U_{k}^{\prime \prime}+8 U_{k}^{(3)} \phi^{2} .
\end{aligned}
$$

The symmetrized expression for the bosonic loop functions $J_{\alpha \beta}^{B}\left(p_{0}\right)$ is given by Eq. B15 where $\alpha, \beta \in\{\sigma, \pi\}$. The fermionic loop functions $J_{\alpha}^{F}\left(p_{0}\right)$ can be cast in the form

$$
\begin{aligned}
& J_{\pi}^{F}\left(p_{0}\right)=h^{2}\left(J_{0}^{F}\left(p_{0}\right)-m_{\psi}^{2} J_{1}^{F}\left(p_{0}\right)\right), \\
& J_{\sigma}^{F}\left(p_{0}\right)=h^{2}\left(J_{0}^{F}\left(p_{0}\right)+3 m_{\psi}^{2} J_{1}^{F}\left(p_{0}\right)\right),
\end{aligned}
$$

where explicit expressions for $J_{0}^{F}\left(p_{0}\right)$ and $J_{1}^{F}\left(p_{0}\right)$ are given by Eqs. (B16)- B17).

$$
\begin{aligned}
& J_{\alpha \beta}^{B}\left(p_{0}\right)+J_{\beta \alpha}^{B}\left(p_{0}\right)=\frac{k^{4}}{3 \pi^{2}}\left(\left[1+n_{B}\left(E_{\alpha}\right)+n_{B}\left(E_{\beta}\right)\right] \frac{\left(E_{\alpha}+E_{\beta}\right)^{3}\left(E_{\alpha}^{2}+E_{\alpha} E_{\beta}+E_{\beta}^{2}\right)+\left(E_{\alpha}^{3}+E_{\beta}^{3}\right) \cdot p_{0}^{2}}{4 E_{\alpha}^{3} E_{\beta}^{3}\left(p_{0}^{2}+\left(E_{\alpha}+E_{\beta}\right)^{2}\right)^{2}}\right. \\
& -\left[n_{B}\left(E_{\alpha}\right)-n_{B}\left(E_{\beta}\right)\right] \frac{\left(E_{\alpha}-E_{\beta}\right)^{3}\left(E_{\alpha}^{2}-E_{\alpha} E_{\beta}+E_{\beta}^{2}\right)+\left(E_{\alpha}^{3}-E_{\beta}^{3}\right) \cdot p_{0}^{2}}{4 E_{\alpha}^{3} E_{\beta}^{3}\left(p_{0}^{2}+\left(E_{\alpha}-E_{\beta}\right)^{2}\right)^{2}} \\
& -\left[n_{B}^{\prime}\left(E_{\alpha}\right)+n_{B}^{\prime}\left(E_{\beta}\right)\right] \frac{\left(E_{\alpha}^{2}-E_{\beta}^{2}\right)^{2}+\left(E_{\alpha}^{2}+E_{\beta}^{2}\right) p_{0}^{2}}{4 E_{\alpha}^{2} E_{\beta}^{2}\left(p_{0}^{2}+\left(E_{\alpha}-E_{\beta}\right)^{2}\right)\left(p_{0}^{2}+\left(E_{\alpha}+E_{\beta}\right)^{2}\right)} \\
& \left.+\left[n_{B}^{\prime}\left(E_{\alpha}\right)-n_{B}^{\prime}\left(E_{\beta}\right)\right] \frac{\left(E_{\alpha}^{2}-E_{\beta}^{2}\right)\left(E_{\alpha}^{2}+E_{\beta}^{2}+p_{0}^{2}\right)}{4 E_{\alpha}^{2} E_{\beta}^{2}\left(p_{0}^{2}+\left(E_{\alpha}-E_{\beta}\right)^{2}\right)\left(p_{0}^{2}+\left(E_{\alpha}+E_{\beta}\right)^{2}\right)}\right) \\
& J_{0}^{F}\left(p_{0}\right)=\frac{k^{4}}{6 \pi^{2}}\left(\left[1-n_{F}\left(E_{\psi}-\mu\right)-n_{F}\left(E_{\psi}+\mu\right)\right] \frac{-4 E_{\psi}^{2}\left(E_{\psi}^{2}+3 k^{2}\right)+\left(5 E_{\psi}^{2}-k^{2}\right) p_{0}^{2}}{E_{\psi}^{3}\left(4 E_{\psi}^{2}+p_{0}^{2}\right)^{2}}\right. \\
& \left.-n_{F}^{\prime}\left(E_{\psi}-\mu\right) \frac{E_{\psi}^{2}-k^{2}+2 E_{\psi} \mathrm{i} p_{0}}{E_{\psi}^{2}\left(2 E_{\psi}+\mathrm{i} p_{0}\right) \mathrm{i} p_{0}}+n_{F}^{\prime}\left(E_{\psi}+\mu\right) \frac{E_{\psi}^{2}-k^{2}-2 E_{\psi} \mathrm{i} p_{0}}{E_{\psi}^{2}\left(2 E_{\psi}-\mathrm{i} p_{0}\right) \mathrm{i} p_{0}}\right) \\
& J_{1}^{F}\left(p_{0}\right)=\frac{k^{4}}{6 \pi^{2}}\left(\left[1-n_{F}\left(E_{\psi}-\mu\right)-n_{F}\left(E_{\psi}+\mu\right)\right] \frac{12 E_{\psi}^{2}+p_{0}^{2}}{E_{\psi}^{3}\left(4 E_{\psi}^{2}+p_{0}^{2}\right)^{2}}\right. \\
& \left.-n_{F}^{\prime}\left(E_{\psi}-\mu\right) \frac{1}{E_{\psi}^{2}\left(2 E_{\psi}+\mathrm{i} p_{0}\right) \mathrm{i} p_{0}}+n_{F}^{\prime}\left(E_{\psi}+\mu\right) \frac{1}{E_{\psi}^{2}\left(2 E_{\psi}-\mathrm{i} p_{0}\right) \mathrm{i} p_{0}}\right)
\end{aligned}
$$

[1] T. Schäfer and D. Teaney, Rept.Prog.Phys. 72, 126001 (2009), 0904.3107.

[2] P. B. Arnold, G. D. Moore, and L. G. Yaffe, JHEP 0011, 001 (2000), hep-ph/0010177.

[3] H. B. Meyer, Eur.Phys.J. A47, 86 (2011), 1104.3708.

[4] E. Taylor and M. Randeria, Phys.Rev. A81, 053610 (2010), 1002.0869.

[5] H. B. Meyer, JHEP 0808, 031 (2008), 0806.3914.

[6] C. Shen, U. Heinz, P. Huovinen, and H. Song, Phys.Rev. C84, 044903 (2011), 1105.3226.

[7] P. Kovtun, D. Son, and A. Starinets, Phys.Rev.Lett. 94, 111601 (2005), hep-th/0405231.

[8] G. Aarts and J. M. Martinez Resco, JHEP 0204, 053 (2002), hep-ph/0203177.
[9] M. Haas, L. Fister, and J. M. Pawlowski, (2013), 1308.4960.

[10] O. Philipsen, Acta Phys.Polon.Supp. 5, 825 (2012), 1111.5370.

[11] J. Berges, N. Tetradis, and C. Wetterich, Phys.Rept. 363, 223 (2002), hep-ph/0005122.

[12] J. Polonyi, Central Eur.J.Phys. 1, 1 (2003), hepth/0110026.

[13] J. M. Pawlowski, Annals Phys. 322, 2831 (2007), hepth/0512261.

[14] B.-J. Schaefer and J. Wambach, Phys.Part.Nucl. 39, 1025 (2008), hep-ph/0611191.

[15] H. Gies, Lect. Notes Phys. 852, 287 (2012), hep$\mathrm{ph} / 0611146$. 
[16] P. Kopietz, L. Bartosch, and F. Schutz, Lect.Notes Phys. 798, 1 (2010).

[17] J. Braun, J.Phys. G39, 033001 (2012), 1108.4449.

[18] G. Baym and N. D. Mermin, J. Math. Phys. 2, 232 (1961).

[19] N. Landsman and C. van Weert, Phys.Rept. 145, 141 (1987).

[20] M. Jarrell and J. Gubernatis, Physics Reports 269, 133 (1996).

[21] M. Asakawa, T. Hatsuda, and Y. Nakahara, Prog.Part.Nucl.Phys. 46, 459 (2001), hep-lat/0011040.

[22] H. J. Vidberg and J. W. Serene, Journal of Low Temperature Physics 29, 179 (1977).

[23] D. Dudal, O. Oliveira, and P. J. Silva, (2013), 1310.4069.

[24] N. Strodthoff, B.-J. Schaefer, and L. von Smekal, Phys.Rev. D85, 074007 (2012), 1112.5401.

[25] K. Kamikado, N. Strodthoff, L. von Smekal, and J. Wambach, Phys.Lett. B718, 1044 (2013), 1207.0400.

[26] S. Floerchinger, JHEP 1205, 021 (2012), 1112.4374.

[27] A. K. Das, Finite temperature field theory (World Scientific, 1997).

[28] S. Strauss, C. S. Fischer, and C. Kellermann, Phys.Rev.Lett. 109, 252001 (2012), 1208.6239.

[29] D. Nickel, Annals Phys. 322, 1949 (2007), hep$\mathrm{ph} / 0607224$.

[30] J. A. Mueller, C. S. Fischer, and D. Nickel, Eur.Phys.J. C70, 1037 (2010), 1009.3762.

[31] S.-x. Qin and D. H. Rischke, Phys.Rev. D88, 056007 (2013), 1304.6547.

[32] K. Kamikado, N. Strodthoff, L. von Smekal, and J. Wambach, (2013), 1302.6199.

[33] D. Jungnickel and C. Wetterich, Phys.Rev. D53, 5142 (1996), hep-ph/9505267.

[34] B.-J. Schaefer and J. Wambach, Nucl. Phys. A757, 479 (2005), nucl-th/0403039.

[35] J. Braun, L. M. Haas, F. Marhauser, and J. M. Pawlowski, Phys.Rev.Lett. 106, 022002 (2011).

[36] C. S. Fischer, J. Luecker, and J. A. Mueller, Phys.Lett. B702, 438 (2011), 1104.1564.

[37] C. S. Fischer and J. Luecker, Phys.Lett. B718, 1036 (2013), 1206.5191.

[38] J. Luecker, C. S. Fischer, L. Fister, and J. M. Pawlowski, PoS CPOD2013, 057 (2013), 1308.4509.
[39] L. M. Haas, R. Stiele, J. Braun, J. M. Pawlowski, and J. Schaffner-Bielich, Phys.Rev. D87, 076004 (2013), 1302.1993.

[40] T. K. Herbst, M. Mitter, J. M. Pawlowski, B.-J. Schaefer, and R. Stiele, (2013), 1308.3621.

[41] C. Wetterich, Phys. Lett. B301, 90 (1993).

[42] J.-P. Blaizot, R. Mendez-Galain, and N. Wschebor, Phys.Rev. E74, 051116 (2006), hep-th/0512317.

[43] J.-P. Blaizot, R. Mendez-Galain, and N. Wschebor, Phys.Rev. E74, 051117 (2006), hep-th/0603163.

[44] M. Le Bellac, Thermal Field Theory (Cambridge University Press, 1996).

[45] D. F. Litim, Phys. Rev. D64, 105007 (2001), hepth/0103195.

[46] F. Karsch, E. Laermann, P. Petreczky, S. Stickan, and I. Wetzorke, Phys.Lett. B530, 147 (2002), heplat/0110208.

[47] S. Datta, F. Karsch, P. Petreczky, and I. Wetzorke, Phys.Rev. D69, 094507 (2004), hep-lat/0312037.

[48] H. Ding et al., Phys.Rev. D86, 014509 (2012), 1204.4945.

[49] R. Schmidt and T. Enss, Phys. Rev. A 83, 063620 (2011), 1104.1379.

[50] N. Dupuis, Phys. Rev. A 80, 043627 (2009), 0907.2779.

[51] A. Sinner, N. Hasselmann, and P. Kopietz, Phys. Rev. Lett. 102, 120601 (2009), 0811.0624.

[52] J. Braun, K. Schwenzer, and H.-J. Pirner, Phys. Rev. D70, 085016 (2004), hep-ph/0312277.

[53] T. K. Herbst, J. M. Pawlowski, and B.-J. Schaefer, Phys. Lett. B696, 58 (2011), 1008.0081.

[54] N. Strodthoff and L. von Smekal, (2013), 1306.2897.

[55] B.-J. Schaefer and J. Wambach, Phys. Rev. D75, 085015 (2007), hep-ph/0603256.

[56] R.-A. Tripolt, J. Braun, B. Klein, and B.-J. Schaefer, (2013), 1308.0164.

[57] T. D. Cohen, Phys.Rev.Lett. 91, 222001 (2003), hep$\mathrm{ph} / 0307089$.

[58] E. E. Svanes and J. O. Andersen, Nucl.Phys. A857, 16 (2011), 1009.0430.

[59] K. Kamikado, N. Strodthoff, L. von Smekal, and J. Wambach, Phys.Lett. B718, 1044 (2013), 1207.0400.

[60] Y. Hidaka, O. Morimatsu, and T. Nishikawa, Phys.Rev. D67, 056004 (2003), hep-ph/0211015. 